COMPUTATIONAL METHODS IN SCIENCE AND TECHNOLOGY 4,71-73 (1998)

\title{
VISUALIZATION OF MONTE CARLO SPIN CLUSTERS FOR THE ISING MODEL IN 2 DIMENSION
}

\author{
L. DĘBSKI AND G. KAMIENIARZ \\ Computational Physics Division, Institute of Physics, Adam Mickiewicz University, \\ ul. Umultowska 85, 61-614 Poznań, Poland, \\ e-mail: $\quad g j k @ p e a r l . a m u . e d u . p l$
}

\begin{abstract}
The Monte Carlo algorithms and the Java applet designed for graphical presentation of spin cluster in the Ising model in two dimensions are presented. The procedure is available in the Division of Computational Physics under the address: http://pearl.amu.edu.pl/DSP.html
\end{abstract}

\section{INTRODUCTION}

The aim of this report is graphical representation of the Ising cluster dynamics dependent on temperature and the simulation algorithm.

We consider the Ising model in two dimensions characterized by the hamiltonian

$$
H=-J \sum_{\langle i, j} \boldsymbol{s}_{i} \boldsymbol{s}_{j}
$$

where $J$ stands for the coupling, the spin variable $s_{i}= \pm 1$ and the sum runs over the neighbouring pairs. We define the reduced temperature $t=T / T_{c}$, where

$$
\frac{k_{B} T_{C}}{J}=\frac{2}{\ln (1+\sqrt{2})}
$$

and impose $0 \leq t \leq 10$. The variable $t$ is called "temp" in our programme. We generate the spin configuration according to the Metropolis [1], Swendsen-Wang [2] and Wolff [3] algorithms.

The Metropolis algorithm is a single spin-flip algorithm. Swendsen-Wang and Wolffs algorithm are called cluster algorithms. Cluster algorithm can dramatically reduce the effects of the critical slowing down in the Monte Carlo simulations [3-5],

\section{THE METHODS OF VISUALIZATION}

The Monte Carlo method for sampling of the Gibbs distribution involves an element of chance $[6,7]$. Random (or, more strictly, pseudorandom) numbers are used to select the configurations of the system. The most important and most frequently used algorithm for the Markov process is that invented by Metropolis et al. in 1953. The change in the energy of the system consequent upon the change from configuration $\alpha$ to $\alpha^{\prime}$, is calculated. If the energy change is negative, then the new configuration is automatically accepted, if, however, it is positive, the new configuration is accepted with the probability $\mathrm{e}^{-\beta\left(E_{\alpha^{\prime}}-E_{\alpha}\right)}$.

The Swendsen-Wang algorithm was introduced in 1987. The essential idea was to let the system itself decide the shape of the "clusters" of spins which are to be flipped simultaneously. 
The scheme of Swendsen-Wang algorithm is:

- take a spin configuration and form a network of bonds by joining together all neighbours aligned,

- keep these bonds with the probability $1-e^{4 \beta J}$, or delete them with the probability $e^{4 \beta J}$. This step will cut most of large spins clusters into two or more smaller clusters,

- treat the spin orientations of the new, smaller, clusters as the basic units and orient them randomly, i. e., up or down with equal probability,

- finally, reconstruct the original lattice of spins from the reoriented clusters.

The whole procedure is taken as a single Monte Carlo evolution step for the system.

Wolff (1989) has inverted an algorithm cluster, similar in spirit to that of Swendsen and Wang, which eliminates the problem of critical slowing down completely.

The spin configurations generated by the Monte Carlo algorithms are illustrated in the Java applet available in the author's web page. As shown in Fig. 1, the web page includes three boards with the labels describing the relevant algorithm at the top of the window. The boards are arranged vertically on the screen, below them there are the buttons: START, STOP and KROK, as well as the text field with the inscription: "Twoja bieżąca temperatura" (Your current temperature). The boards are composed of 32 squares which change the colour depending on the spin orientation. The white colour stands for $s=-1$ while blue, green or pink stand for $s=+1$.

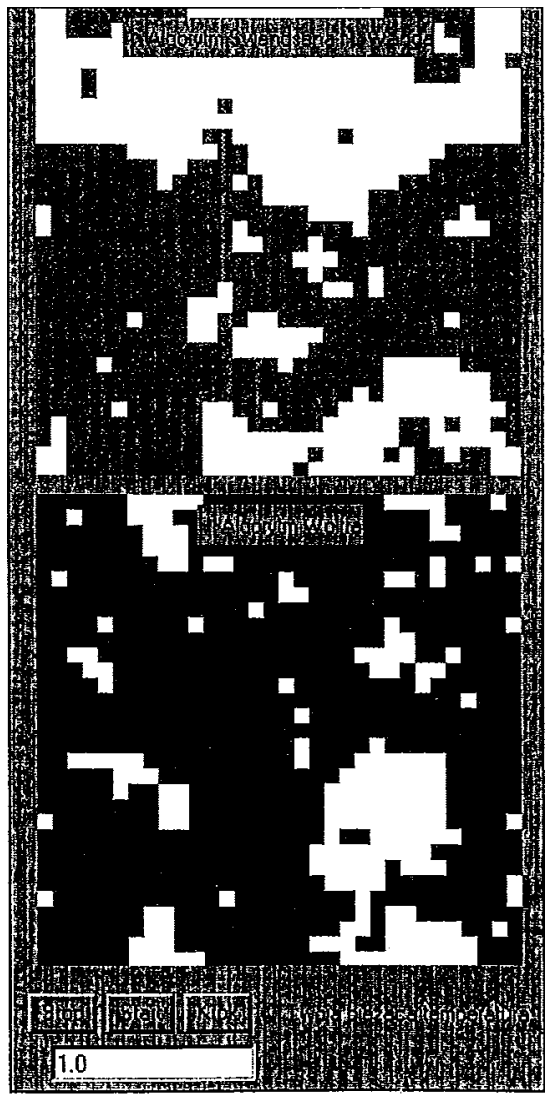

Fig. 1. Two lattices of the Ising spin configurations generated by the cluster algorithms with labels and buttons 


\section{DESCRIPTION OF THE MONTE CARLO APPLET}

This applet runs automatically when you surf to web page under the address: http://pearl. amu.edu.pl./DCP.html. On this page, there are three windows in three different colors. Below the windows, there are three buttons: start, stop, krok and text field.

Button "START" - when pressed/clicked - start the applet. All boards will change colour.

Button "STOP" - when pressed/clicked - stops the program.

Button "KROK" - when pressed/clicked - initializes one step of the applet. Only one spin configuration will be changed and repainted in the board.

In text field you can set your temperature from 0.0 to 10.0000000 . The critical temperature corresponds to the variable temp. $=1.0$. When the temperature is out of range, you will see the text: "Podałeś złą temperaturę" (Wrong choice); when you take a temperature from within this range, you will see on the screen: "Podałeś dobra temperaturę" (right choice). Next press button ENTER on the keyboard. The temperature will be loaded and the three boards will be repainted. The applet starts.

\section{Acknowledgment}

The authors wish to acknowledge the access to Poznań Supercomputing and Networking Center and partial financial support from the State Committee for Scientific Research within the grant 8T11F015 09 .

\section{References}

[1] N. Metropolis, A. W. Rosenbluth, M. N. Rosenbluth, A. H. Teller, J. Chem. Phys. 21, 1087(1953)

[2] R. H. Swendsen and J.-S Wang, Phys. Rev. Lett. 58, 86 (1987)

[3] U. Wolff, Phys. Rev. Lett. 62, 361 (1989)

[4] A. M. Ferrenberg, D. P. Laudau, Phys. Rev. B44, 5081 (1991)

[5] H. W. J. Blote, G. Kamieniarz, Physica A196, (1993)

[6] P. Pawlicki, G. Kamienniarz, L. Dębski, Physica A242, 290 (1997)

[7] P. Kozłowski, P. Pawlicki, G. Kamieniarz, Computational Methods in Science and Technology, 3, 39 (1997) 